\title{
AUTONOMÍA DE LA VOLUNTAD Y GESTACIÓN POR SUSTITUCIÓN EN ARGENTINA*
}

\author{
AUTONOMY OF THE WILL AND GESTATIONAL SURROGACY IN \\ ARGENTINA
}

PATRicio Jesús CURTi**

\begin{abstract}
Resumen: La gestación por sustitución es una realidad que, si bien no se encuentra regulada legalmente en Argentina, actualmente, ha sido receptada favorablemente y de manera unánime por la jurisprudencia local. La autonomía de la voluntad como una cuestión central de esta práctica es una de las mayores preocupaciones que giran en torno a la construcción de las decisiones judiciales, visibilizándose todo tipo de argumentos al respecto que permiten elaborar una serie de premisas para entender mejor a esta técnica de reproducción humana asistida.

Palabras clave: Gestación por sustitución, autonomía de la voluntad, Argentina, jurisprudencia, maternidad subrogada.

Abstract: Gestational surrogacy is a reality which, although it is not legally regulated in Argentina, nowadays, it has been accepted favourably and unanimously by local jurisprudence. Autonomy of the will as a central issue of this practice is one of the major concerns that revolve around the construction of judicial decisions, revealing an array of arguments in this regard that allow for a series of premises to be developed in order to better understand this technique of assisted human reproduction.
\end{abstract}

Keywords: Gestational surrogacy, autonomy of the will, Argentina, jurisprudence, surrogate motherhood.

SUMARIO: I. PALABRAS INTRODUCTORIAS; II. DE LO GLOBAL A LO PARTICULAR: EL CONTEXTO REGIONAL COMO PUNTO DE PARTIDA; III. ARGENTINA: LA FUERZA DE LA REALIDAD; IV. AUTONOMÍA DE LA VOLUNTAD Y ANALISIS DE LA CASUISTICA ARGENTINA; V. COLOFÓN; VI. BIBLIOGRAFÍA.

* http://doi.org/10.15366/rjuam2018.38.006

Fecha de recepción: 6 de noviembre de 2018.

Fecha de aceptación: 19 de diciembre de 2018.

** Abogado. Maestrando de la Maestría de Familia, Infancia y Adolescencia, con tesis en elaboración (Universidad de Buenos Aires, Argentina). Docente de Familia y Sucesiones (Universidad de Buenos Aires, Argentina). Miembro del Proyecto de Investigación UBACyT «Hacia una ley especial sobre técnicas de reproducción humana asistida. Bioética, derechos humanos y familias» (Universidad de Buenos Aires, Argentina). Miembro del Proyecto de Investigación en Maestría (Universidad de Buenos Aires, Argentina). Dirección de correo electrónico de contacto: patriciojcurti@gmail.com. 


\section{PALABRAS INTRODUCTORIAS}

La revolución reproductiva a la que se enfrenta el mundo actual exige un abordaje que involucre varios ejes que la atraviesan. Focalizando en la gestación por sustitución (en adelante, GS), uno de los aspectos fundamentales, se vincula estrechamente con la autonomía de la voluntad, jugando un papel vital.

En primer lugar, para llegar a trazar algunas reflexiones sobre esta cuestión dentro del territorio argentino, habrá que posicionarse en la región para referenciar la realidad local en función de lo que sucede en los países aledaños.

En segunda instancia, se abordará la situación legal en la que se encuentra la GS en el país, con una breve reseña de su historia normativa y el estado actual, anticipando que el vacío legal sobre el tema es el problema y la solución, ante la complejidad que suscita el silencio de la ley.

Justamente por esta deuda que presenta el Derecho de las familias se ha generado una gran actividad judicial al respecto, corrigiendo esta falencia y posibilitando que esta práctica tenga receptividad. A través del amplio bagaje analítico que ofrecen estas decisiones jurisdiccionales se pueden trazar varias conclusiones. Aunque, a los efectos del tema tratado se extraerán los argumentos subyacentes que posibilitarán una mejor comprensión del papel que juega la autonomía de la voluntad dentro de la concreción de la GS. Veamos.

\section{DE LO GLOBAL A LO PARTICULAR: EL CONTEXTO REGIONAL COMO PUNTO DE PARTIDA}

A los fines de dimensionar la situación de la GS en el campo del Derecho argentino resulta importante comenzar con una aproximación general, tomando como muestra algunos países de la zona que admiten esta práctica.

Por ejemplo, Brasil, que bajo la modalidad altruista y gracias a la jurisprudencia, dio a conocer el primer caso en el que una niña fue inscrita como hija de dos progenitores y habiendo sido gestada por la prima de uno de ellos, mediante transferencia de un embrión compuesto por el óvulo de una donante y esperma de uno de ellos. El juez de la ciudad de Recife, terminó autorizando la inscripción señalada ${ }^{1}$. A su vez, en el año 2015, mediante Resolución 2121 del Consejo Federal de Medicina², se determinó que la GS sólo podría llevarse a cabo cuando exista un problema médico por parte de la mujer a la que se le impida o contraindique que sea ella quien lleve adelante la gestación y extendiendo la aplicación

\footnotetext{
1 Caso por «processo de indicação de paternidade». Requirientes «M. A. A. e W. A. A.» de 28 de febrero de 2012. Juízo de Direito da $1^{\text {a }}$ Vara de Família e Registro Civil da Comarca do Recife.

2 Brasil. Conselho Federal de Medicina. Resolução CFM nº 2.121, de 16 de julho de 2015. Disponible en $<$ http://bit.ly/1NW9tTQ>. [Consultado el 10/10/2018].
} 
a uniones homoafectivas. Por otro lado, establece la gratuidad con que debe efectuarse la referida técnica y que la gestante deberá ser pariente, hasta el cuarto grado -inclusive- de alguna de las personas interesadas en ser progenitores ${ }^{3}$.

Siguiendo con los países donde se encuentra permitida la GS, hay que destacar a Uruguay, donde existe la permisión legal a través de la Ley que regula las técnicas de reproducción humana asistida núm. 9.167 y contempla la posibilidad solo en mujeres cuyo útero no pueda gestar un embarazo debido a enfermedades genéticas o adquiridas, quienes podrán acordar con un familiar suyo de segundo grado de consanguinidad, o de su pareja -en su caso-, la implantación y gestación del embrión propio. Se prevé la suscripción del acuerdo por todas las partes intervinientes y bajo la naturaleza gratuita. Para garantizar el acceso por parte de las personas interesadas a esta práctica, el Decreto nacional núm. 84/15 en su artículo 2 incorpora a la GS como una técnica de reproducción humana asistida de alta complejidad con cobertura a cargo del Fondo Nacional de Recursos.

Por su parte, Perú no cuenta con una ley que regule la GS, pero se han suscitado dos casos en las cuales la justicia dio respuesta favorable a este procedimiento. En el primero de ellos - de 06/01/20094- un matrimonio que deseaba tener hijos, aunque ella padecía una insuficiencia renal grave e hipertensión arterial que la pondría en riesgo de vida ante un embarazo. De esta manera, recurrieron a las técnicas de fecundación in vitro utilizando su propio material genético y contando con el apoyo de la madre de la señora, quien decidió llevar adelante la gestación de su nieta. Al nacer la niña, fue inscrita en la clínica como hija del matrimonio a través de una acción de impugnación de la maternidad de la pareja. En el segundo de los $\operatorname{casos}^{5}$-sin entrar en detalles casuísticos- se acogió la filiación en favor de los requirentes. El principal argumento de la sentencia recayó en la inexistencia de razones para que el Estado -actuando a través del Juzgado Constitucional- niegue la protección que el ordenamiento convencional reconoce y dado que no hay legislación que prohíba expresamente la técnica de reproducción utilizada por los actores.

También cabe mencionar a México, donde en términos generales la GS está prohibida, pero hay dos Estados donde legalmente se encuentra permitida: Tabasco y Sinaloa. Más allá de esta permisión, es importante destacar lo dicho por la Sala Primera de la Suprema Corte de Justicia de México, el 27/01/2017:

\footnotetext{
3 Para comprender con mayor exactitud lo establecido en la Resolución 2121/2015, se recomienda ahondar en DA SILVA GALLO, J. y LETTIERI GRACINDO, G., «Reproducción asistida, derecho de todos. ¿Y el registro del hijo? ¿Cómo proceder?», Rev. bioét., vol. 24-2, 2016, pp. 250 a 259. Disponible en <http://dx.doi. org/10.1590/1983-80422016242125>. [Consultado el 13/10/2018].

4 Juzgado Especializado de Familia núm. 15, 06/01/2009, «C. M. S. A. v. L. A. U. O. y otros / impugnación de maternidad» (inédito).

5 Corte Superior de Justicia de Lima, Quinto Juzgado Especializado en lo Constitucional, 21/02/2017 (inédito).
} 
«A partir de las consideraciones del Tribunal Europeo de Derechos Humanos sobre la similitud entre las parejas homosexuales y heterosexuales en cuanto a su capacidad de desarrollar una vida familiar, la Primera Sala de esta Suprema Corte de Justicia de la Nación entiende que la vida familiar entre personas del mismo sexo no se limita únicamente a la vida en pareja, sino que puede extenderse a la procreación y a la crianza de niños y niñas según la decisión de los padres. Así, existen parejas del mismo sexo que hacen vida familiar con niños y niñas procreados o adoptados por alguno de ellos, o parejas que utilizan los medios derivados de los avances científicos para procrear» ${ }^{6}$.

Con respecto al Estado de Tabasco, se establece en el artículo 92 del Código Civil que

«[e]n el caso de los hijos nacidos como resultado de la participación de una madre gestante sustituta, se presumirá la maternidad de la madre contratante que la presenta, ya que este hecho implica su aceptación. En los casos en los que participe una madre subrogada, deberá estarse a lo ordenado para la adopción plena. Se entiende por madre gestante sustituta, la mujer que lleva el embarazo a término y proporciona el componente para la gestación, más no el componente genético. Por el contrario, la madre subrogada provee ambos: el material genético y el gestante para la reproducción. Se considera madre contratante a la mujer que convenga en utilizar los servicios de la madre gestante sustituta o de la madre subrogada, según sea el caso. Salvo el caso de que se trate de un hijo nacido de una madre gestante sustituta, cuando el hijo nazca de una mujer casada que viva con su esposo, el Oficial del Registro Civil no podrá asentar como padre a otro que no sea el mismo marido, excepto que éste haya desconocido al hijo y exista sentencia ejecutoria que así lo declare».

Por otra parte, el Estado de Sinaloa, en el artículo 283 y siguientes del Código Familiar se contempla específicamente que el acuerdo sobre GS podrá ser oneroso o gratuito. Las dos modalidades establecen requisitos para la mujer gestante: su edad, que debe oscilar entre 25 y 30 años, buena salud, entre otras; y los médicos e instituciones deben estar autorizados por la Secretaría de Salud del Gobierno del Estado. Estos sistemas de tinte más liberal cuentan con la particularidad de que la filiación se establece sin intermediaciones, es decir, directamente entre el niño o niña y los requirentes, no teniendo estos que someterse a procedimientos como la adopción, por ejemplo.

En Chile, actualmente, no existe ninguna legislación específica sobre GS, aunque el 29/11/2017, se presentó ante el Parlamento de ese país un proyecto de ley para permitirla y su regulación giraría en aspectos centrales tales como: el acceso a la práctica para personas solas y parejas -heterosexuales y homosexuales- que podrían estar casadas o no, y el

\footnotetext{
6 Semanario Judicial de la Federación, Suprema Corte de Justicia de México. Disponible en

$<$ http://sjf.scjn.gob.mx/SJFSem/Paginas/DetalleGeneralV2.aspx?Epoca=\&Apendice=\&Expresion=\&Dom inio $=$ Tesis $\% 20 \% 20$ publicadas $\% 20 \mathrm{el} \% 20$ viernes $\%>$. [Consultado el 23/10/2018].
} 
carácter altruista y solidario de la gestante, que no recibiría dinero más allá del necesario para cubrir los gastos directamente derivados del embarazo.

\section{ARGENTINA: LA FUERZA DE LA REALIDAD}

Luego del recorrido trazado, que muestra la tendencia hacia la aceptación de este tipo de Técnica de Reproducción Humana Asistida (TRHA), llegamos a Argentina que, marcando como punto de partida el año 2012, muestra cuatro momentos definidos.

El camino se inicia con el Decreto presidencial núm. 191, de 23/02/2011, mediante el que se crea la «Comisión para la Elaboración del Proyecto de Ley de Reforma, Actualización y Unificación de los Códigos Civil y Comercial de la Nación». Luego de varios meses de debate y tras la consulta a 109 juristas, el Anteproyecto es elevado al Poder Ejecutivo en el mes de febrero del año 2012. Posteriormente, en junio de ese año se envió a ambas Cámaras del Congreso y bajo la indicación de que sea creada en ese ámbito una «Comisión Bicameral para la Elaboración del Proyecto de Ley de Reforma, Actualización y Unificación de los Códigos Civil y Comercial de la Nación» que se abocaría a trabajar sobre la nueva normativa.

En noviembre de 2013 se emitió un dictamen de mayoría y cinco de minoría que fue remitido a la Cámara de Senadores de la Nación para tratarlo inmediatamente en una sesión especial, en la que se aceptaron algunos de los cambios propuestos, entre ellos, la eliminación de la regulación de la GS ${ }^{7}$. El texto aprobado por la Cámara de Senadores fue girado a la Cámara de Diputados y al año siguiente con esta particular modificación fue aprobado, entrando en vigor el 01/08/2015.

En cuanto a la figura en cuestión, este hito en el Derecho de las familias dejó su impronta -pese a la supresión- marcando una directriz que la jurisprudencia tomó, bajo un supuesto de índole constitucional ${ }^{8}$ : sin estar prohibida, la GS en Argentina está permitida.

El dictamen de la Comisión Bicameral eliminó la GS del texto definitivo del Código Civil y Comercial. Para ello, se sostuvo lo siguiente: «[s]e suprime la gestación por sustitución por los motivos que se explican a continuación. En su reemplazo se propone el desdoblamiento del texto del artículo 560 de manera que el primer párrafo pasa a ser el nuevo artículo 560 y el segundo párrafo queda como texto del nuevo artículo 561 . La gestación por sustitución es la figura jurídica dentro del Libro Segundo sobre Relaciones de familia que más voces encontradas ha generado. Sucede que es un proceso especial de técnicas de reproducción asistida que compromete a tres personas y no a dos, para alcanzar la maternidad/paternidad. Es decir, una tercera persona con quien no se tendrá vínculo filial alguno. La especialidad y mayor complejidad de esta técnica de reproducción humana deriva del propio texto legal proyectado, siendo este tipo de práctica médica la única que involucraba un proceso judicial previo con la previsión de cumplir varios elementos o requisitos para la viabilidad de la acción judicial. En este sentido, la gestación por sustitución encierra dilemas éticos y jurídicos de gran envergadura que ameritarían un debate más profundo de carácter interdisciplinario. En este contexto de incertidumbre y cuasi silencio legal en el Derecho Comparado, se propone de manera precautoria, eliminar la gestación por sustitución del Proyecto de reforma».

8 El artículo 19 de la Constitución Nacional argentina, dispone: «[1]as acciones privadas de los hombres que de ningún modo ofendan al orden y a la moral pública, ni perjudiquen a un tercero, están sólo reservadas 
Ante este panorama, la pregunta a realizarse es: ¿De qué forma se regulaba la GS en el Anteproyecto de Reforma del Código Civil y Comercial? Se preveía en el artículo 562 de la siguiente manera:

«El consentimiento previo, informado y libre de todas las partes intervinientes en el proceso de gestación por sustitución debe ajustarse a lo previsto por este Código y la ley especial. La filiación queda establecida entre el niño nacido y el o los comitentes ${ }^{9}$ mediante la prueba del nacimiento, la identidad del o los comitentes y el consentimiento debidamente homologado por autoridad judicial. El juez debe homologar solo si, además de los requisitos que prevea la ley especial, se acredita que: a) se ha tenido en miras el interés superior del niño que pueda nacer; b) la gestante tiene plena capacidad, buena salud física y psíquica; c) al menos uno de los comitentes ha aportado sus gametos; d) el o los comitentes poseen imposibilidad de concebir o de llevar un embarazo a término; e) la gestante no ha aportado sus gametos; f) la gestante no ha recibido retribución; $g$ ) la gestante no se ha sometido a un proceso de gestación por sustitución más de dos (2) veces; h) la gestante ha dado a luz, al menos, un (1) hijo propio. Los centros de salud no pueden proceder a la transferencia embrionaria en la gestante sin la autorización judicial. Si se carece de autorización judicial previa, la filiación se determina por las reglas de la filiación por naturaleza».

Luego de la entrada en vigor del Código Civil y Comercial de la Nación (CCyC) se presentaron varios proyectos de ley para regular la GS. Sin entrar en un análisis de cada uno de ellos y en líneas generales, es importante resaltar que mantienen los predicamentos de la norma proyectada; siendo siete los que actualmente se encuentran con estado parlamentario ${ }^{10}$.

Delineados los periodos legislativos, resta señalar que la falta de regulación de la GS no fue obstáculo para que se presentasen planteos judiciales que encierran diferentes situaciones de GS, buscando, básicamente, solucionar la cuestión filial luego o previamente al nacimiento del niño o niña.

La supresión legal y la carencia de normas prohibitivas implican que el tema quede sujeto a la discrecionalidad judicial, aunque vale decir que lo que preveía el Proyecto de Reforma del CCyC marcaba un parámetro de funcionamiento acorde al sistema constitucionalconvencional y la judicialización obligó a los jueces a tener en cuenta esos lineamientos, para resolver los conflictos suscitados.

\footnotetext{
a Dios, y exentas de la autoridad de los magistrados. Ningún habitante de la Nación será obligado a hacer lo que no manda la ley, ni privado de lo que ella no prohíbe».

9 En cuanto a este término, es preciso señalar que a partir de las conclusiones elaboradas por la Comisión 2 del Congreso Internacional de Derecho de las Familias celebrado en el mes de septiembre de 2018 en Mendoza (Argentina) se estableció el uso de "requirente/s" y no "comitente/s" (como lo preveía el Proyecto de Reforma del Código Civil y Comercial).

10 A modo ilustrativo se detallan: 3202-D-2017, 3765-D-2017, 5141-D-2017. 0084-D-2018, 0630-D-2018 y D-1374-D-2018.
} 
Hasta ahora la jurisprudencia -de manera unánime- ha consolidado la aceptación de GS por cuanto todas sus decisiones fueron favorables; razón por la que revaloriza o reafirma la importancia de que el Congreso de la Nación sancione una ley especial ${ }^{11}$. En este sentido se ha dicho: «Funcionando como el piso mínimo de garantía o protección a considerar en los casos en que se presenta una práctica de gestación por sustitución mientras no exista regulación legal al respecto y mientras no se expida el Congreso a través de una ley que prohíba o regule la gestación por sustitución, seremos los jueces los encargados de resolver los casos en que se solicite la autorización o se lleve a cabo la práctica y en esa tarea no se podrá consentir o convalidar ninguna situación que se encuentre "por debajo" de la regulación que aquél proyecto de reforma pretendió establecer» ${ }^{12}$.

Ahora bien, al no contar con normas específicas que regulen la GS, la forma de resolver estos casos se da con el actual artículo 562 del CCyC, referido a la voluntad procreacional, que establece «[1] os nacidos por las técnicas de reproducción humana asistida son hijos de quien dio a luz y del hombre o de la mujer que también ha prestado su consentimiento previo, informado y libre en los términos de los artículos 560 y 561 , debidamente inscripto en el Registro del Estado Civil y Capacidad de las Personas, con independencia de quién haya aportado los gametos».

La solución que más se ajusta a la realidad es la declaración de inconstitucionalidad del citado artículo -en cuanto a que determina que

«quien da a luz» es progenitor/a del/la niño/a que nació producto de esta TRHA-, basando la decisión en argumentos constitucionales-convencionales, que se vinculan con el derecho a la identidad, entre otros. Si bien se sabe que «la declaración de inconstitucionalidad constituye un remedio de ultima ratio que debe evitarse, de ser posible, mediante una interpretación del texto legal en juego compatible con la Ley Fundamental, pues siempre debe estarse a favor de la validez de las normas, lo cierto es que la claridad meridional del artículo 562 del CCiv. y Com es tal que resulta dificultoso compatibilizarla con el ordenamiento jurídico sistémico, es decir, de acuerdo a las fuentes de interpretación previstas en el título preliminar, en particular, con los tratados de derechos humanos, sin tachar su letra de constitucional» ${ }^{13}$.

\footnotetext{
$11 \mathrm{Al}$ respecto, se ha dicho: «es preciso legislar de modo que cuando nazca un niño pueda ser inmediatamente inscripto como hijo de quienes quieren ser sus progenitores o progenitor conforme al elemento volitivo expresado, ya que el interés superior del niño comprometido merece ser atendido con la máxima diligencia y premura» (Juzgado Familia núm. 7 de Lomas de Zamora, «H. M. Y OTRO/A S/MEDIDAS PRECAUTORIAS - art.232 del CPCC-», 30/12/2015).

Juzgado de Familia núm. 2 de Mendoza, 6/9/2017 (inédito).

13 DE LA TORRE, N., «La gestación por sustitución "hecha en casa": el primer reconocimiento jurisprudencial en parejas del mismo sexo», Derecho de Familia. Revista Interdisciplinaria de Doctrina y Jurisprudencia, vol. 1, 2017, p. 134.
} 
Nombrando el primero de los casos que se ha dado en el país (bajo esta línea) el 30/12/2015, la Jueza titular del Juzgado de Familia núm. 7 de Lomas de Zamora, explicó que

«es inconstitucional y anticonvencional, en este caso concreto, en cuanto no reconoce la maternidad de la mujer que ha expresado su voluntad procreacional mediante el consentimiento informado, sino la de la mujer gestante, lo que implica que de no declararse la inconstitucionalidad de la norma -atento su imposible compatibilización con el ordenamiento jurídico de acuerdo a una interpretación literal, teleológica y sistémica, como lo establece en el articulado del Título Preliminar del Código de fondo, y atento que no puede obviarse la relevancia del artículo por formar parte de las reglas generales en materia de TRHA-, la niña por nacer habría de ser inscripta como hija de su tía (que además será su madrina), hermana de sus primas y primo, y sobrina de sus padres, lo que resulta incompatible con el deber de garantizar el ejercicio de los derechos humanos comprometidos en la causa por parte de la jurisdicción, máxime cuanto las personas que recurren a los estrados judiciales en la búsqueda de una respuesta titularizan por su situación de vulnerabilidad una protección diferencial (art. 75 incs. 22 y 23 de la Constitución Nacional, arts. 1 y 2 Convención Americana de Derechos Humanos)» ${ }^{14}$.

Sin embargo, para algunos autores, la declaración de inconstitucionalidad de la mencionada norma no haría falta, porque con la interpretación y el dinamismo de los arts. 1 y 2 del CCyC se llegaría al mismo resultado. Este argumento se apoya en que, tal como se ha sostenido: «[s]i el modelo constitucional y convencional de derecho nos informa que debeos resolver apelando a un diálogo entre fuentes, entendemos que al mismo resuelvo, se podía llegar, sin recurrir a lo que la Corte ha definido como "la última ratio". Asimismo, consideramos que admitir esta posibilidad es abrir las puertas a un mecanismo que impactará negativamente en el funcionamiento del actual Código, por cuanto significará parte del contenido de un sistema que recién está empezando a funcionar» ${ }^{15}$.

En definitiva, las respuestas que se han vislumbrado para rellenar el silencio legislativo son, por un lado, la declaración de inconstitucionalidad del art. $562 \mathrm{y}$, por el otro, la interpretación y dinamismo que brindan los arts. 1 y 2 del CCyC. En concreto, hay que decir que, a fecha de hoy ${ }^{16}$, se han dictado treinta sentencias nacionales -partiendo de casos resueltos por operadores judiciales de Argentina, suscitados en el referido territorio y entre argentinos- que han acogido favorablemente a la GS. Seis de ellas han sido anteriores a

\footnotetext{
14 Juzgado Familia núm. 7 de Lomas de Zamora, «H. M. Y OTRO/A S/MEDIDAS PRECAUTORIAS -art. 232 del CPCC-», cit.

15 KRASNOW, A. N. «Los principios de pluralidad, autonomía y solidaridad familiar en la filiación», en KRASNOW, A. N. e IGLESIAS, M. (dirs.), Derecho de las familias, Rosario (Nova Tesis), 2016, p. 256.

16 Fecha de referencia: 15/10/2018.
} 
la entrada en vigor del $\mathrm{CCyC}$, y las veinticuatro restantes se dictaron con posterioridad al cambio normativo.

\section{AUTONOMÍA DE LA VOLUNTAD Y ANÁlisis de LA CASUÍSTICA ARGENTINA}

Centrándonos en el tema convocante conviene empezar este apartado con algunos cuestionamientos que giran a su alrededor: ¿Cuáles son los límites a la autonomía de la voluntad la/s parte/s requirente/s? ¿Presta la persona gestante un consentimiento verdaderamente informado? ¿Debe aceptarse que sea practicada con fines comerciales o sólo con fines altruistas? ¿Tiene que existir algún tipo de vínculo familiar o afectivo entre las partes?

Luego de un análisis de las sentencias dictadas en el país, es posible aseverar que, entre las fundamentaciones tenidas en cuenta al momento de resolver favorablemente los casos planteados, en mayor o menor medida se abordó a la «autonomía de las personas intervinientes» como un argumento relevante para resolver el conflicto planteado en sede judicial.

Para una mejor comprensión del tema, hay que destacar que lo afirmado anteriormente tiene dos bastiones: por un lado, la/s persona/s requirente/s y por el otro, la persona gestante. De manera ejemplificativa e ilustrativa se han compilado algunas consideraciones de la jurisprudencia:

a) Requirente/s:

- Derecho a la vida privada, vinculado con la autonomía reproductiva y el acceso a servicios de salud reproductiva. Respeto a la forma en que las personas desean tener hijos/as y el tipo familiar que desean constituir. Se tomaron en cuenta - principalmente y entre otraslas directrices de la Corte Interamericana de Derechos Humanos (CIDH) del último caso vinculado con el tema («Artavia Murillo y otros (F.I.V) vs. Costa Rica», del 28/11/2012):

«El derecho de toda persona de organizar, con arreglo a la ley, su vida individual y social conforme a sus propias opciones y convicciones, resultando un derecho humano básico, que abarca el principio de dignidad, autonomía personal, desarrollo personal y el derecho a establecer y desarrollar relaciones con otros seres humanos y con el mundo exterior [...]. En referencia al caso concreto, considera que se trata de una combinación particular de diferentes aspectos de la vida privada, que se relacionan con el derecho a fundar una familia, el derecho a la integridad física y mental, y específicamente los derechos reproductivos de las personas $[\ldots]$ el artículo 17 de la Convención Americana de Derechos Humanos reconoce el papel central de la familia y la vida familiar en la existencia de una persona y en la sociedad en general $»^{17}$.

17 Tribunal de Familia núm. 7 de Rosario, 02/12/2014. Dato de publicación: RDF 2016-III, 135. 
También se señaló:

«La libertad, definida así, es un derecho humano básico, propio de los atributos de la persona, que se proyecta en toda la Convención Americana. Asimismo, la Corte ha resaltado el concepto de libertad y la posibilidad de todo ser humano de autodeterminarse y escoger libremente las opciones y circunstancias que le dan sentido a su existencia, conforme a sus propias opciones y convicciones [...]. La protección a la vida privada abarca una serie de factores relacionados con la dignidad del individuo, incluyendo, por ejemplo, la capacidad para desarrollar la propia personalidad y aspiraciones, determinar su propia identidad y definir sus propias relaciones personales» ${ }^{18}$.

Resulta interesante la conceptualización del límite a la autonomía de las personas requirentes que se ha desarrollado en relación a los casos resueltos: «La libertad de intimidad o autonomía de la voluntad implica "poder de decisión", cuyo único límite es el considerar si tal decisión conlleva la consumación de un daño directo a los derechos fundamentales de otra persona» ${ }^{19} ; \mathrm{y}$ «existe una creciente concientización de que en una sociedad liberal la gente debería elegir dentro de sus posibilidades, la forma en que desean tener hijos y el tipo de familia que quieren constituir, y no deberían ser penalizados por ello. Mientras el modelo de familia elegido no produzca un daño obvio y directo a los niños nacidos de tales acuerdos (o a la mujer implicada) y se garantice el derecho del niño a la información sobre las circunstancias de su procreación, la ley no debería prohibirla» ${ }^{20}$.

En último término, poniendo en hechos estas valoraciones normativas y subjetivando la cuestión se ha dicho: «[1]a gestación por sustitución, de acuerdo a la diversidad funcional de la progenitora, constituye para esta pareja la única oportunidad real de ejercer su derecho fundamental a formar una familia y de ejercer una maternidad y una paternidad responsables y en igualdad de condiciones que los demás» ${ }^{21}$.

- Igualdad y no discriminación. Se ha puesto de resalto la difícil interacción que se produce en una sociedad que exalta la maternidad y paternidad como valores, pero rechaza u obstaculiza los medios científicos para lograrla cuando no se obtiene naturalmente, respondiendo a causas estructurales, económicas, de salud, etc.; cayendo necesariamente en un trato que atenta contra estos principios constitucionales-convencionales. Particularmente, se reseñó: «se encuentra en pugna su derecho a la igualdad y a la no discriminación ya que se les debe posibilitar el acceso a las técnicas de reproducción humana asistida en

\footnotetext{
Juzgado de Familia núm. 2 de Mendoza, 06/09/2017, cit.

Juzgado Nacional en lo Civil núm. 8, 20/09/2016. Dato de publicación: elDial.com - AA9A82.

Juzgado de Familia de Gualeguay, 19/11/2013. Dato de publicación: LA LEY 2013-D, 195.

${ }^{21}$ Juzgado Familia núm. 7 de Lomas de Zamora, 30/11/2016. Cita online: AR/JUR/85614/2016.
} 
similares condiciones que, a otras personas, que también tienen limitaciones para lograr un embarazo» ${ }^{22}$.

Críticamente, de manera explícita y concluyente -sin más agregados que realizar-se puso en manifiesto una situación que se suscita a nivel mundial: «[d]ejemos de ser hipócritas, hace muchos años que nacen en el exterior niños que son hijos de padres argentinos, utilizando métodos científicos y legales fácilmente utilizables en nuestro suelo» ${ }^{23}$.

- Presencia de la voluntad procreacional. En cuanto a este eje, hay que decir que, tal como lo refirió la jurisprudencia: «en las técnicas de reproducción humana asistida es la voluntad procreacional el elemento central y fundante para la determinación de la filiación, con total independencia de si el material genético pertenece a las personas que, efectivamente, tienen la voluntad de ser padres o madres [...]. Definitivamente rige aquí el principio de la autonomía de la voluntad y no la del puro dato genético, para dar nacimiento al vínculo filial $»^{24}$. Asimismo, en esta línea: «a la hora de pensar la paternidad/maternidad, coloca en primer plano la voluntad procreacional que no es más ni menos que la faz volitiva y subjetiva de los protagonistas ${ }^{25}$.

En cuanto a la voluntad procreacional (vinculada con la GS) se la entendió

«como el deseo o intención de crear una nueva vida, derecho que también merece la protección del ordenamiento jurídico al tutelarse a la persona en su decisión libre de tener un hijo [...]. Estas nuevas formas de concebir la familia requieren sean reconocidos sus derechos filiatorios. Así se expuso con claridad la existencia de uniones afectivas donde la reproducción natural no resulta posible, obligan admitir la construcción de un parentesco que no se funde en bases biológicas, sino en la construcción de vínculos basados en la "socioafectividad"; y cuya construcción dependen de la existencia de una voluntad procreacional, a la que sin duda debe dar una respuesta el ordenamiento jurídico $»^{26}$.

b) Gestante:

- ¿Explotación de las mujeres? (pobres por las ricas). Se observa este cuestionamiento por parte de los jueces que han intervenido, en torno al análisis casuístico. Lo que se teme avalar con la resolución judicial tiene que ver con la postura doctrinal que afirma:

\footnotetext{
22 Juzgado de Familia núm. 3 de Córdoba, 22/11/2017 (inédito).

23 Juzgado de Familia núm. 1 de Mendoza, 29/7/2015. Disponible en $<$ http://www.colectivoderechofamilia. com/fa-pcial-juz-flia-no1-mendoza-gestacion-por-sustitucion/>.

24 Tribunal de Familia núm. 7 de Rosario; 02/12/2014, cit.

25 Tribunal de Familia núm. 7 de Rosario; 02/12/2014, cit.

26 Juzgado de Familia núm. 2 de Mendoza, 15/12/2015. Dato de publicación: RDF 2016-III, 113.
} 
«La gestación por sustitución supone una explotación de la mujer debido a que conlleva a la utilización de las mujeres pobres por las ricas o, como consecuencia del turismo reproductivo, que se verá luego, a la utilización de las mujeres de los países del tercer mundo o periféricos, por las mujeres de países del primer mundo. Sin perjuicio de que también puede conllevar a situaciones de abuso respecto de el o los comitentes, generalmente “desesperados" por tener un hijo» ${ }^{27}$.

En contra de esta presunción se han alzado diversas voces que se volcaron en la jurisprudencia sobre GS:

«Para refutar el argumento de la explotación o cosificación de la mujer gestante, entiende que tratándose de un acuerdo voluntario y libre no hay porqué hablar de explotación, ni aun interviniendo dinero. El argumento de la explotación es paternalista y subestima la capacidad de consentir de la mujer. Para algunos, incluso, el hecho de que la gestación por sustitución sea comercial tampoco justifica que la subrogación deba prohibirse o que los contratos de gestación por sustitución no puedan ser ejecutables. Se entiende que el argumento de la cosificación o comercio priva a la mujer del derecho a la privacidad y autodeterminación y las trata injustamente» ${ }^{28}$.

Con base en lo anterior, parece que la única salida para evitar la situación consiste en realizar determinadas comprobaciones, como, por ejemplo: «[e]n el supuesto en estudio, la teoría de la explotación o cosificación de la mujer gestante queda desvirtuada al tratarse de un acuerdo voluntario y libre, que al no conllevar un interés económico por tener su base en el vínculo afectivo de las partes» ${ }^{29}$; «También debe descartarse el concepto de "explotación" de la madre sustituta, ya que el objetivo de la gestante es totalmente altruista, oficiando de vehículo para que el deseo de los futuros padres pueda hacerse realidad» ${ }^{30}$; o bien: «La motivación de la Sra. xxx se vincula al deseo de ayudar a la pareja para la conformación de una familia, con el nacimiento de un hijo. No se detectan intereses económicos, ni gananciales ligados a provecho propio» ${ }^{31}$.

Es notoria la importancia que se le otorga a este tema. Hasta se ha vislumbrado en muchos fallos una exhortación implícita al resto de los poderes:

«Una buena regulación puede ser un instrumento eficaz para impedir la formación de un verdadero "mercado negro de vientres" en el que la mujer es un objeto usado por personas que desean tener un hijo a cualquier costa. La posible explotación

\footnotetext{
Juzgado de Familia de Gualeguay, 19/11/2013, cit. Juzgado de Familia de Gualeguay, 19/11/2013, cit.

Juzgado Nacional en lo Civil núm. 102, 18/05/2015. Dato de publicación: LA LEY 2015-C, 522.

Juzgado de Familia núm. 5 de Viedma, 07/07/2017 (inédito).

31 Juzgado de Familia núm. 2 de Mendoza, 06/09/2017, cit.
} 
de mujeres que se encuentran en situaciones desventajosas es una preocupación legítima, pero la prohibición o el silencio de la ley lo potencia y, consecuentemente, aumenta el número de mujeres que pueden sufrir explotación como consecuencia de esta práctica. En otras palabras, la falta de regulación o la prohibición legal provocan que esta técnica se realice al margen de la ley y, en muchos casos, en la clandestinidad, lo que incrementa los posibles abusos e injusticias. Regular implica que debe hacerse dentro de ciertos márgenes que disminuyen la posibilidad de abusos e injusticias» ${ }^{32}$.

- Manipulación del cuerpo de la persona. Cosificación: falta de consentimiento libre. A los fines de desvirtuar eventuales afirmaciones doctrinales que son evaluadas en la mayoría de los decisorios, tales como la siguiente: «se sostiene que la gestación por sustitución importa una cosificación de la mujer en virtud de que la gestante se convierte en un mero "ambiente" o "incubadora humana" para el hijo de otro. Esta cosificación, por un lado, atenta contra la libertad y autonomía de las mujeres debido a que éstas no consienten libremente ${ }^{33}$, se traen argumentos opuestos para desvirtuar el alcance de esta postura. En este sentido, la interdisciplina se hace notoriamente presente para relevar datos que extingan esta posibilidad:

«[n]o presenta condicionamientos psicopatológicos que le impidan comprender los alcances de asumir la gestación de un embrión ajeno (fs. xxx). En el mismo sentido el psicólogo xxx informa que la señora $\mathrm{xxx}$ se encuentra bien orientada en tiempo y espacio, presenta estado anímico sin depresión ni ansiedad, no se observan alteraciones en la sensopercepción, teniendo plena conciencia sobre las implicancias de ser gestante en el proceso de subrogación de vientre, como así también que en el momento tras el parto el bebé será dado a los señores xxx $\mathrm{y} \mathrm{xxx}{ }^{34}$. «Me encuentro totalmente segura y firme en la decisión. No recibí retribución económica para prestarme a la gestación, lo hago por un acto de amor hacia el matrimonio. Mi madre, luego de tenerme a mí, no pudo tener más hijos, existe una similitud en lo que le sucedió a mi madre y lo que les sucede a xxx y xxx. Yo quedé como única hija, pero lo que le ocurre a este matrimonio es aún peor, comprendo cabalmente lo que les sucede y quiero ayudarlos. No aporté gametos para la formación de embriones crioconservados, y en ningún otro, sólo tengo a mi hija xxx de xx años (fs. $\mathrm{xxx})\rangle^{35}$.

Incluso, se ha caído en la comprobación de los ítems que traía el Anteproyecto de Reforma del CCyC, mediante los cuales se chequeaba que no hubiese un consentimiento doblegado por diversos factores: «[e]n autos se encuentra acreditado: la gestante se encuentra

\footnotetext{
Juzgado de Familia núm. 1 de Mendoza, 29/7/2015, cit.

Juzgado de Familia de Gualeguay, 19/11/2013, cit.

34 Tribunal de Familia núm. 7 de Rosario, 02/12/2014, cit.

35 Tribunal de Familia núm. 7 de Rosario, 02/12/2014, cit.
} 
en buen estado de salud física y psíquica conforme evaluación de la Junta Transdiciplinaria (fs. $\mathrm{xxx}$ ), informe psicológico (fs. xxx), certificado ginecológico (fs. $\mathrm{xxx}$ ) [...] la gestante no ha recibido retribución económica, conforme lo manifestado a fs. xxx, acordando el pago de la cobertura de la obra social, alimentación y obtención de cremas para el cuidado de la piel (fs. $\mathrm{xxx}) »^{36}$.

También se ha dicho, entre otras valoraciones: «la mujer gestante obró con pleno y libre consentimiento por cuestiones de solidaridad y humanismo decidió, con el asentimiento de su marido, llevar adelante el embarazo para dar una vida hacia sus amigos que naturalmente no pueden tener hijos, fines que son acordes a los requisitos que exigía el proyecto ${ }^{37}$. A su vez, a través de un informe agregado al expediente-que fue volcado en una sentencia- quedó expresado:

«en la entrevista personal se pudo constatar personalmente su pleno consentimiento y capacidad. Demostró absoluta comprensión del proceso y de sus acciones, con un grado importante de compromiso. Debo dejar constancia que culminé la entrevista gratamente sorprendida en cuanto a la posibilidad de corroborar fácticamente lo que hace tiempo venimos sosteniendo: Como personas y mujeres, existe de forma básica y fundamental un derecho de las gestantes a servirse libremente de su cuerpo y a tomar decisiones al respecto. Entender que la gestación por sustitución implica siempre explotación de la mujer es un reduccionismo paternalista que subestima a la mujer y a su capacidad de consentir [...]. Una de las principales preocupaciones que se han expresado desde posturas feministas con respecto a la gestación por sustitución son las condiciones de desigualdad de las mujeres gestantes y el efecto que esto puede tener en su capacidad de decidir» ${ }^{38}$.

Asimismo: «[e]stimo que lo que pareciera ser que desde el informe se pretende resguardar, es decir a la gestante, no es más que una desvalorización de xxx, quien de manera clara, específica, concreta y autónoma decide como ella misma lo dijo "alojar por un tiempo al hijo de sus cuñados". Privarla de ello significaría una intromisión impropia en su vida privada que no puede ser consentida, ni tolerada por los órganos jurisdiccionales $\rangle^{39}$.

- Ausencia de voluntad procreacional. Como contrapartida a la presencia de lo volitivo en el caso de los requirentes, se analiza -desde la autonomía de la voluntad como punto de partida- la carencia de este elemento en cabeza de la persona que gesta. En este orden de ideas, los magistrados intervinientes se han abocado a comprobar la falta voluntad para ejercer la función parental. De manera muy ilustrativa se ha dicho, por ejemplo: «xxx a través del presente quiere hacer constar que conoce específicamente el procedimiento de

\footnotetext{
36 Tribunal de Familia núm. 7 de Rosario, 02/12/2014, cit.

37 Tribunal de Familia núm. 5 de Rosario, 27/05/2016. Dato de publicación: RDF 2016-IV, 131.

38 Juzgado de Familia núm. 2 de Mendoza, 06/09/2017, cit.

39 Juzgado de Familia núm. 3 de Córdoba, 22/11/2017, cit.
} 
transferencia de embriones que se le realizó; y como tal reconoce y acepta que no hay vinculación genética alguna con los embriones transferidos ni tampoco voluntad procreacional» ${ }^{40}$.

Rotundamente se expresó la jurisprudencia, diciendo:

«Resultaría contradictorio aplicar la regla del artículo 562 a los casos de gestación por sustitución, cuando el propio sistema jurídico del mismo Libro Segundo, Título V, reconoce a la voluntad procreacional como determinante del vínculo filial, a más de implicar directamente una grave vulneración al derecho a la filiación del niño o niña que como consecuencia de este tipo de técnicas nazca, al obligarlo/a a mantener una filiación materna con quien no ha querido asumir ese rol, habiéndolo manifestado previo y fehacientemente, privándolo del verdadero vínculo filiatorio y de su derecho a la identidad subjetiva. Asimismo, constituiría una grave vulneración a los derechos de la mujer no gestante que hubiera otorgado su voluntad procreacional, en un claro trato desigualitario y discriminatorio desconociéndole los derechos que la propia ley del mismo cuerpo normativo le otorga. Como también implicaría una seria afectación al derecho a la libertad y privacidad de la mujer gestante, obligándola a ser madre cuando precisamente su voluntad es de no serlo» ${ }^{41}$.

Luego de la recopilación vista anteriormente y desechando la noción errónea que presume a la gestante como un sujeto en condiciones de vulnerabilidad, es posible afirmar que el equilibrio entre quienes intervienen en la GS está dado por la limitación de la autonomía impuesta a la/s persona/s requirente/s, que se funda en la protección hacia la persona que gesta.

En este orden de ideas, resulta importante realizar una valoración: si los temores ante la permisión de la GS giran principalmente en torno a la vulneración de derechos de la persona gestante, sin vaivenes se puede afirmar que el Proyecto de Reforma del CCyC contrarrestaba esta posibilidad, poniendo la mirada en aspectos centrales, que -inclusiveha marcado rotundamente la doctrina. En este sentido se ha dicho:

«I) Consentimiento de la gestante: indispensable para comprobar certeramente que la gestante presta su consentimiento libre, pleno e informado al sometimiento de esta práctica. Libre, en tanto debe ser prestado sin coerción, violencia, intimidación ni presiones de ningún tipo. Pleno, pues debe ser prestado por alguien capaz de consentir a todas las cuestiones implicadas. Informado, tanto en los aspectos médicos como en los psicológicos, todo lo cual supone asesoramiento adecuado, en un contexto en el que las nociones de especialidad e interdisciplina resultan trascendentales en toda práctica médica, especialmente en las TRHA. II) Plena

40 Juzgado Nacional en lo Civil núm. 7, 15/06/2016 (inédito).

41 Juzgado de Familia de Maipú (Mendoza), 04/04/2018 (inédito). 
capacidad, buena salud física y psíquica de la gestante: un requisito que garantiza que el consentimiento emana de una mujer competente, con autonomía plena, y que la GS no redundará en perjuicio de su salud. Para la comprobación de esta exigencia, la mujer debe someterse a estudios físicos y psíquicos previos. Además de esas certificaciones médicas y psicológicas previas, el juez toma la decisión auxiliado por un equipo interdisciplinario que evalúa la salud física y psíquica de la gestante y dictamina sobre su aptitud para actuar como tal. III) Se autoriza sólo la sustitución gestacional: la gestante sólo aporta la gestación, no sus óvulos. IV) La gestante no se ha sometido a un proceso de GS más de una determinada cantidad de veces: este requisito tiende a evitar abusos y que mujeres se conviertan en "máquinas productoras de hijos ajenos". Es decir, previene la "cosificación de la gestante". También se justifica a los efectos de evitar la profesionalización de esta práctica. Se trata de una solución equilibrada, que por un lado garantiza la libertad, y por el otro, protege a las mujeres cuando la autonomía no es tal. V) La gestante debe tener al menos un hijo previo propio: este requisito asegura que la gestante comprende la gravedad de su compromiso, porque ella ha sufrido los rigores de la gestación y el parto de un niño; garantiza, pues, que presta un consentimiento verdaderamente informado. Solo la mujer que ha gestado y ha dado a luz a un niño puede saber lo que ese acontecimiento implica e irroga, desde el punto de vista físico, médico y psicológico. La gestante debe ser protegida desde que la práctica implica, además de la cesión de su útero, la disposición, aunque sea temporal, de su integridad psicofísica. VI) Otra cuestión central, tiene que ver con prever y proyectar un formato legal que regule compensaciones - no retribuciones-que sean fijadas por la autoridad de aplicación que al prever criterios uniformes no importe un enriquecimiento de la persona gestante, evitando de esta manera que esta sea la motivación y consecuentemente reducir así la tan temida “explotación”» ${ }^{42}$.

Siguiendo con el análisis de la jurisprudencia y a los fines de esbozar otra conclusión sobre la autonomía de la voluntad en la GS, hay que subrayar que desde el primero hasta el último caso que llegó a la instancia judicial se han utilizado diversas estrategias jurídicas para conseguir el resultado querido. Algunos se encausaron procesalmente como procesos autosatisfactivos ${ }^{43}$, mientras que otros trascurrieron por los canales propios del trámite cautelar (buscando, a veces, impedir la inscripción del nacimiento por imperio del art. 562 $\mathrm{CCyC)}$ y los de las acciones declarativas.

\footnotetext{
42 LAMM, E., «Gestación por sustitución. Una valiente y valiosa sentencia», LA LEY 21/12/2015, 1. Cita online: AR/DOC/4185/2015.

43 Es decir, aquellos de carácter urgente, autónomos, dictados, en principio, inaudita parte y que responden a una situación que requiere necesariamente una imperiosa solución prescindiendo de un proceso principal y en los cuales se hace prevalecer el principio de celeridad, que obliga a reducir la cognición y a postergar la bilateralidad, con el fin de otorgar una tutela eficaz y rápida.
} 
Por último, varios debieron acceder a las vías impugnativas de la filiación de la maternidad y, claro -como ya se dijo- están los que no encontraron otro camino que la declaración de inconstitucionalidad.

Para una mejor comprensión, se puede trazar una división de los momentos en que fueron planteadas las diversas tácticas utilizadas y haciendo un recuento de las veces que se utilizaron cada una de ellas:

1) Luego del nacimiento: impedir provisoriamente la inscripción y solicitarla a favor de los requirentes ( 2 veces), medida autosatisfactiva, acción declarativa de certeza ${ }^{44}$ y amparo solicitando la inscripción (7 veces) e impugnación de la maternidad (9 veces).

2) Antes del nacimiento: autorización judicial previa (12 veces).

Con base en lo expuesto, se puede reflexionar acerca de otra cuestión que se enfrenta en la praxis, en miras de la protección de las personas involucradas en la GS. Posteriormente al nacimiento, cualquier análisis que se baraje respecto de la vulneración de derechos de los adultos implicados se torna abstracto. Al confrontar las condiciones en las que se produjo la práctica con el conocido interés superior del niño, sin dudas, no cabe posibilidad alguna de corregir los posibles atropellos a los derechos de la persona gestante o de las requirentes. Si el niño o niña nació, la respuesta judicial no puede dejar de brindarle un resguardo legal a esa realidad. Definitivamente, es lo correcto en este momento, ya que cualquier decisión jurisdiccional queda condicionada al principio rector señalado.

Pero, a los fines de amparar la real autonomía de la voluntad de quienes intervienen en la GS -principalmente de la persona gestante, quien tiene mayores posibilidades de situarse en una posición desfavorable- la estrategia que mejor preserva los derechos de todas las partes -incluso del niño o niña-es la que se plantea la intervención judicial previa a la transferencia embrionaria en el útero de la persona que llevará a cabo la GS.

Este es el procedimiento que preveía el Proyecto de Reforma del CCyC protegiendo a todas las partes involucradas. El juez, constatando el cumplimiento de los requisitos protectorios que establecía la norma, homologaba el acuerdo y a partir de ese momento los centros de salud podían proceder a la transferencia del embrión.

En este sentido, vale destacar que a partir del fallo judicial número diecinueve ${ }^{45}$ se ha utilizado principalmente esta estrategia jurídica. Esto no es casual y tiene que ver con dos factores. Por un lado, el andamiaje jurisdiccional que receptaba a la GS como una práctica permitida y, por ello, tornaba inútil acudir a un Juzgado a regularizar la situación, pasando por

44 Tiene que ver con una acción en la que se tiende a obtener una sentencia meramente declarativa, para hacer cesar un estado de incertidumbre sobre la existencia, alcance o modalidades de una relación jurídica, siempre que esa falta de certeza pudiera producir un perjuicio o lesión actual al actor y éste no dispusiera de otro medio legal para ponerle término inmediatamente.

45 Juzgado de Familia núm. 5 de Viedma, cit. 
un tiempo prolongado de incertidumbre y con un/a niño/a que ya había nacido/a. Por el otro, hay que subrayar el trabajo que ha realizado la Comisión Asesora en Técnicas de Reproducción Humana Asistida, un organismo multidisciplinar integrado por los máximos referentes en reproducción asistida de Argentina, que en esa época elaboró una «Guía de Buenas Practicas sobre Gestación por Sustitución en Argentina» y que se alineó con la forma que preveía el Proyecto de Reforma del CCyC, es decir, la autorización judicial pre-transferencia embrionaria.

\section{COLOFÓN}

Se ha tratado de ofrecer un panorama general sobre la realidad local sin perder de vista a la región y principalmente desde una mirada jurisprudencial para culminar en el tema central abordado, con las conclusiones que se han podido efectuar luego del camino recorrido en el país, donde la fuerza de la realidad ha sido elocuente. Aunque es necesario decir algo más: sin ley, la problemática tiende a profundizarse y complejizarse.

No descuidemos un detalle. Los treinta casos que se han resuelto hasta ahora en Argentina tuvieron como protagonistas a personas con un vínculo afectivo previo. Sintéticamente, la persona gestante y los requirentes fueron parientes, amigos o meros conocidos -en porcentajes: $40,75 \%, 40,75 \%$ y $18,50 \%$, respectivamente-. El altruismo y desinterés económico ha sido la «presunción absoluta» para que los jueces destierren cualquier situación de aprovechamiento de una parte con respecto a la otra. Parece que si no hay una prestación monetaria todo funciona «normalmente» en materia de autonomía de la voluntad. Esto no es así, ya que el intercambio monetario no implica necesariamente una mercantilización de seres humanos, ni importa una cosificación o explotación de la persona que ha gestado o del niño/a por nacer, por cuanto puede existir, también, la posibilidad de una «compensación» destinada a cubrir gastos de salud, riesgos, dedicación, tiempos, cuidados, asesoramiento legal y psicológico, lucro cesante e implicancias afectivas del proceso ${ }^{46}$.

Así están dadas las circunstancias. La GS se ha instalado como algo que no puede pensarse que a futuro desaparezca. De nada sirve silenciarla. Pero, además, hay conflictos que hasta la actualidad no se han suscitado, pero anticipadamente no es muy difícil preverlos: ¿Qué pasaría en el caso que la persona gestante decida interrumpir la gestación? ¿Y si lo decidieran los requirentes? ¿Cómo se resolvería un planteo en el que la gestante decide asumir la maternidad de la persona que gestó? ¿Cuáles serían las consecuencias de corroborar los fines comerciales en la GS?, entre otras.

Varios interrogantes. Una respuesta: sin más, urgente regulación.

46 A modo de ejemplo, sirve traer uno de los proyectos de ley -núm. 1374-D-2018- que en su artículo 12 prevé las «compensaciones»: «[e]l acuerdo de gestación por sustitución no puede tener carácter lucrativo o comercial. La compensación económica a cargo de los comitentes y en beneficio de la gestante es válida si sirve para compensar los gastos médicos, de traslados, de asesoramiento legal y psicológico y todos aquellos que sean consecuencia directa de la gestación por sustitución, incluidos los derivados de los tratamientos para provocar el embarazo, el parto y el post parto». 


\section{BIBLIOGRAFÍA}

DA SILVA GALLO, J. y LETTIERI GRACINDO, G., «Reproducción asistida, derecho de todos. ¿Y el registro del hijo? ¿Cómo proceder?», Revista Bioética, vol. 24, núm. 2, 2016. Disponible en <http://dx.doi.org/10.1590/1983-80422016242125>. [Consultado el 13/10/2018].

DE LA TORRE, N., «La gestación por sustitución "hecha en casa”: el primer reconocimiento jurisprudencial en parejas del mismo sexo», Derecho de Familia. Revista Interdisciplinaria de Doctrina y Jurisprudencia, vol. 1, 2017, pp. 134-149.

KRASNOW, A. N. «Los principios de pluralidad, autonomía y solidaridad familiar en la filiación», en KRASNOW, A.N. e IGLESIAS, M. (dirs.), Derecho de las familias, Rosario (Nova Tesis), 2016, pp. 201-222.

LAMM, E., «Gestación por sustitución. Una valiente y valiosa sentencia», LA LEY 21/12/2015, 1, pp. 40-51. Cita online: AR/DOC/4185/2015. 